\title{
Pooling Evidence to Identify Cell Cycle-Regulated Genes
}

\author{
Gaolin Zheng $^{1}$, Tom Milledge ${ }^{1}$, E. Olusegun George ${ }^{2}$, and Giri Narasimhan ${ }^{1}$ \\ ${ }^{1}$ Bioinformatics Research Group (BioRG), School of Computer Science, \\ Florida International University, Miami, Florida, 33199, USA \\ \{gzhen001, tmille01, giri\}@cis.fiu.edu \\ ${ }^{2}$ Department of Mathematical Sciences, University of Memphis, Memphis, TN 38152, USA \\ eogeorge@memphis.edu
}

\begin{abstract}
Most of the biological studies have embraced statistical approaches to make inferences. It is common to have several independent experiments to test the same null hypothesis. The goal of research on pooling evidence is to combine the results of these tests to ask if there is evidence from the collection of studies to reject the null hypothesis. In this study, we evaluated four different pooling techniques (Fisher, Logit, Stouffer and Liptak) to combine the evidence from independent microarray experiments in order to identify cell cycle-regulated genes. We were able to identify a better set of cell cycle-regulated genes using the pooling techniques based on our benchmark study on budding yeast (Saccharomyces cerevisiae). Our gene ontology study on time series data of both the budding yeast and the fission yeast (Schizosaccharomyces pombe) showed that the GO terms that are related to cell cycle are significantly enriched in the cell cycleregulated genes identified using pooling techniques.
\end{abstract}

\section{Introduction}

In bioinformatics analyses, the process of making inferences and extracting knowledge from data is complicated by the fact that the data may arrive from diverse sources. These varied sources of data may be heterogeneous or homogeneous. For example, in order to predict potential transcription factor binding sites, one may use heterogeneous sources of data such as gene expression data (to infer co-expressed genes), ChIP-chip data (to obtain information on binding sites for known transcription factors), upstream promoter sequence data (to infer shared and over-expressed sequence motifs), text literature (to use information from reported biological experiments on known transcription factors and their binding sites), sequence data on other related organisms (to infer conserved regions in the genome sequence and to get supporting evidence for the predictions), gene ontology information (to identify functionally-related genes), and much more. Even for a focused study (with homogeneous sources of data) that attempts to identify which set of genes show significant change in expression in a tissue sample from a lung cancer patient relative to that from a normal patient, it is possible that the data may be collected from a wide variety of individuals and the experiments may be performed by different research groups under varying conditions. While a host of bioinformatics methods exist for making 
inferences from a single data set (along with providing statistical significance for these inferences), a significant challenge is posed when inferences have to be made based on multiple sources of data. It is non-trivial to integrate different sources of data (with different levels of variability and reliability) to arrive at one coherent set of conclusions.

Several procedures for pooling of such evidence in meaningful ways exist in the statistical literature [1-4]. The earliest work on implementing pooling strategies in bioinformatics is to be found in the work of Bailey and Gribskov, where they developed an algorithm called QFAST for the sequence homology search problem [5]. Their algorithm used a variant of Fisher's inverse Chi-square method without reference to the Chi-square distribution for computational efficiency [5]. In this study we investigate and evaluate a variety of statistical techniques to combine or pool together evidence from many homogeneous sources to arrive at meaningful conclusions. To evaluate these techniques, we revisit the familiar problem of using gene expression data to identify cell cycle-regulated genes in two related organisms: budding yeast, Saccharomyces cerevisiae, and fission yeast Schizosaccharomyces pombe. We consider existing tools to identify cell cycle-regulated genes and investigate the change in their performance after they are applied to three different yeast data sets generated by Spellman et al. and the evidence is pooled.

The cell-division cycle is fundamental to the proliferation of all organisms, and knowledge of its regulation helps in understanding various diseases [6]. Periodic gene expression seems to be a universal feature of cell cycle regulation. In 1998, the first genome-wide DNA microarray studies were conducted in the budding yeast, $S$. cerevisiae, to reveal a large number of cell cycle-regulated genes that peaked only once per cell cycle [7, 8]. More recently, microarray experiments have been conducted to study the cell cycle regulation in the fission yeast $S$. pombe, which is a distant relative of budding yeast $[6,9]$. Both $S$. cerevisiae and $S$. pombe are useful organisms for the study of the cell division cycle because they have many well-characterized cell division cycle mutants [10]. In particular, the budding yeast data sets have driven the development of various computational methods for identifying periodically expressed genes [11-15]. Some of these methods even provided statistical significance (in terms of $P$-values) for their predictions $[14,15]$.

In order to demonstrate the efficacy of pooling, we considered two existing tools for identifying cell cycle-regulated genes $[14,15]$. Although several other tools exist for this task, they were not appropriate for our study since they did not return $P$ values for their predictions for the individual experiments.

\section{Methods}

\subsection{Identifying Cell Cycle-Regulated Genes}

We adopted Fisher's $g$-test to test the periodicity of a gene [14]. A $P$-value was obtained for each time course data [14]. The lower the $P$-value, the more periodic is the gene. We also tested the periodicity of a gene using a permutation-based method [15]. 


\subsection{Combining Evidence Using $P$-Values}

Given several independent sets of samples along with the corresponding $P$-values for a certain property, the idea of combining the $P$-values to obtain a unified measure of significance has a long history. The existing methods are Fisher's inverse Chi-square method [1], George and Mudholkar's logit method [2], Stouffer's overall z method [4], and the Liptak-Stouffer [3] method.

In bioinformatics, combining inferences has been applied to sequence homology search, protein classification and scoring motifs $[5,16]$. Their method is a variant of the Fisher's inverse Chi-square method. A more recent application can be found in the work of Oliva et al. on the fission yeast Schizosaccharomyces pombe, where they used Stouffer's method to obtain a single $P$-value for each gene from the three $P$-values inferred from the two elutriation experiments and the cdc25 block-release experiment in order to identify oscillating transcripts [9].

With the goal of evaluating the various $P$-value pooling techniques, we picked the problem of identifying cell cycle-regulated genes in budding yeast and fission yeast. The null hypothesis was that there is no periodic component in the time course data for the gene in question. The first advantage of choosing this problem was the ready availability of several independent cell cycle microarray experiments (alpha, cdc15 and cdc28) to test whether they collectively reject the null hypothesis. The second and more important reason for using the budding yeast data set is that de Lichtenberg et al. had also designed benchmark data to evaluate the results of the predictions [15]. The techniques we evaluate will provide us with $P$-values (for each gene) supporting the claim that the gene in question is cell cycle-regulated. The benchmark data sets allow us to measure the ability of different methods to identify the cell cycle-regulated genes. Below we describe the pooling techniques that were evaluated in this work. Since benchmark data is not readily available for the fission yeast, we were unable to evaluate the data sets in the same way that the budding yeast data set was. We identified the periodically expressed genes in the fission yeast using the pooling methods and the results were evaluated for functional enrichment.

\subsubsection{The Fisher Method}

The Fisher procedure [1] for pooling the $P$-values is given by $F=\sum_{i=1}^{N}-2 \ln \left(P_{i}\right)$

where $\mathrm{N}$ is the number of tests to be combined. Under the null hypothesis, the $P_{\mathrm{i}}$ 's can be assumed to have a uniform distribution in the range of $[0,1]$. Thus under this condition F has a $\chi^{2}$-distribution with $2 \mathrm{~N}$ degrees of freedom. The $P$-value associated with the F-score is given by $P(X \geq F)$, where $X \sim x^{2}(2 N)$. Although Fisher's method is the most commonly used method for combining information across multiple test of the same null hypothesis, one significant drawback of Fisher's method is that it is asymmetrically sensitive to small $P$-values compared to large $P$-values. 


\subsubsection{The Logit Method}

A logit procedure is given by $L=-\sum_{i=1}^{N}\left(P_{i} /\left(1-P_{i}\right)\right)$ [2]. Under the null hypothesis, $L$ has the distribution of a convolution of $\mathrm{N}$ logistic random variables. The distribution of $L$ can be approximated as $L \sim \pi \sqrt{\frac{N(5 N+2)}{3(5 N+4)}} t_{5 N+4}$. The $P$-values associated with $L$ is obtained as $P\left(X>\frac{L}{\pi} \sqrt{\frac{3(5 N+4)}{N(5 N+2)}}\right)$, where, $X$ is a student-t random variable with $5 \mathrm{~N}+4$ degrees of freedom.

\subsubsection{Stouffer's Z-Transform Method}

The Z-transform $Z_{i}=\Phi^{-1}\left(P_{i}\right)$, converts the $P$-values, $P_{\mathrm{i}}$, from each of the $\mathrm{N}$ independent tests into standard norm deviates $Z_{\mathrm{i}}$. The sum of the $Z_{\mathrm{i}}^{\text {' }}$ 's, divided by the square root of the number of tests, $\mathrm{N}$, has a standard normal distribution if the common null hypothesis is true [4]. Thus, $Z_{s}=\sum_{i=1}^{N} Z_{i} / \sqrt{N}$ can be compared to the standard normal distribution to provide a test of the cumulative evidence on the common null hypothesis [4].

\subsubsection{Liptak-Stouffer's Weighted Z-Method}

Liptak generalized Stouffer's Z-transform method by giving different weights to each study according to their power [3]. The scoring is given by $Z_{w}=\sum_{i=1}^{N} W_{i} Z_{i} / \sqrt{\sum_{i=1}^{N} W_{i}^{2}}$. There are different ways to choose the weights. Usually sample size or the inverse of the sample error variance is chosen as the weight for each test. It is debatable whether the weighted Z-method is appropriate since the $P$-values are already weighted by the sample size.

\subsection{Gene Expression Data and Benchmark Data}

We used three budding yeast cell cycle gene expression data. These three gene expression data set were obtained from samples of budding yeast cultures synchronized by three different methods: $\alpha$-factor arrest, and arrest of two different temperature-sensitive mutants (cdc15 and cdc28) [8].

Fission yeast cell cycle data were obtained from $S$. pombe Functional Genomics website [http://www.sanger.ac.uk/PostGenomics/S_pombe/projects/cellcycle/]. Three elutriation and three cdc 25 block-release cell cycle data were used in this study. The goal of using the fission yeast data was to be able to compare the results obtained from the budding and the fission yeast. Plus, fission yeast data sets are very new and substantial[9]. 
The performance of the $P$-value pooling methods were evaluated by measuring their abilities to identify genes from two of the three benchmark sets provided by de Lichtenberg et al. [15]. Benchmark data sets B1 and B2 were used in this comparative study. B1 consisted of a total of 113 genes previously identified as periodically expressed in small-scale experiments [15]. B2 consisted of genes whose promoters were bound by at least one of the nine known cell cycle transcription factors in the Chromatin IP studies [15]. After removing 50 genes that belonged to B1, B2 consisted of 352 genes which were deemed to be cell cycle-regulated. Benchmark data B3 was not used because it was deemed unreliable by the authors [15].

\section{Results and Discussion}

Summarizing our benchmarking experiments on $S$. cerevisiae, we have two methods to identify cell cycle-regulated genes from a single data set (Fisher's $g$-test and permutation method), three different gene expression data sets for $S$. cerevisiae (alpha, cdc15 and cdc28), four methods to pool $P$-values when any given method is used to identify the periodic genes from the three data sets, and two different benchmark sets of known cell cycle-regulated genes (B1 and B2 for $S$. cerevisiae). For $S$. pombe, we have six independent gene expression data sets and no readily available benchmark sets of cell cycle-regulated genes. Finally, for each of the experiments, the lists of significantly periodic genes obtained from the budding and the fission yeast data sets were queried against Gene Ontology database to obtain a list of significantly enriched GO terms.

\subsection{Benchmark Results for S. cerevisiae}

A set of $P$-values were obtained for each data set using either Fisher's $g$-test or permutation method. Each gene was associated with a $P$-value obtained from the individual data sets. The $P$-values were combined using the four $P$-value pooling methods mentioned above for $S$. cerevisiae. For each method, the genes were ranked based on the corresponding $P$-values. Lower $P$-values were ranked higher. Figure 1 shows the performance of Fisher's $g$-test and permuation method on each of three individual microarray expression data sets for $S$. cerevisiae along with that of the four $P$-value pooling techniques to combine the evidence from the three experimental data sets.

\subsection{Function Enrichment of Periodically Expressed Genes}

To assess the ability of pooling technique to identify cell cycle-regulated genes, gene ontology information was used to evaluate whether the identified cell cycle- regulated genes have significant enrichment of one or more terms that are related to cell cycle. This was done using GOMiner [17]. A Fisher's exact test is used to test if a GO term is enriched or depleted. For the budding yeast, three $P$-values were generated for each gene based on the three experiments using Fisher's $g$-test [14]. A single $P$-value was obtained for each gene using the logit pooling procedure. (Only the logit method was used in this analysis, since the four pooling methods were comparable in performance, with the logit method being marginally better). To correct the $P$-values for multiple testing, the method of Benjamini and Hochberg [18] was used to control the 
false discovery rate (FDR). The FDR method controls the expected portion of false positives at a given rate $\mathrm{q}$. A list of 1686 genes was identified as cell cycle-coupled
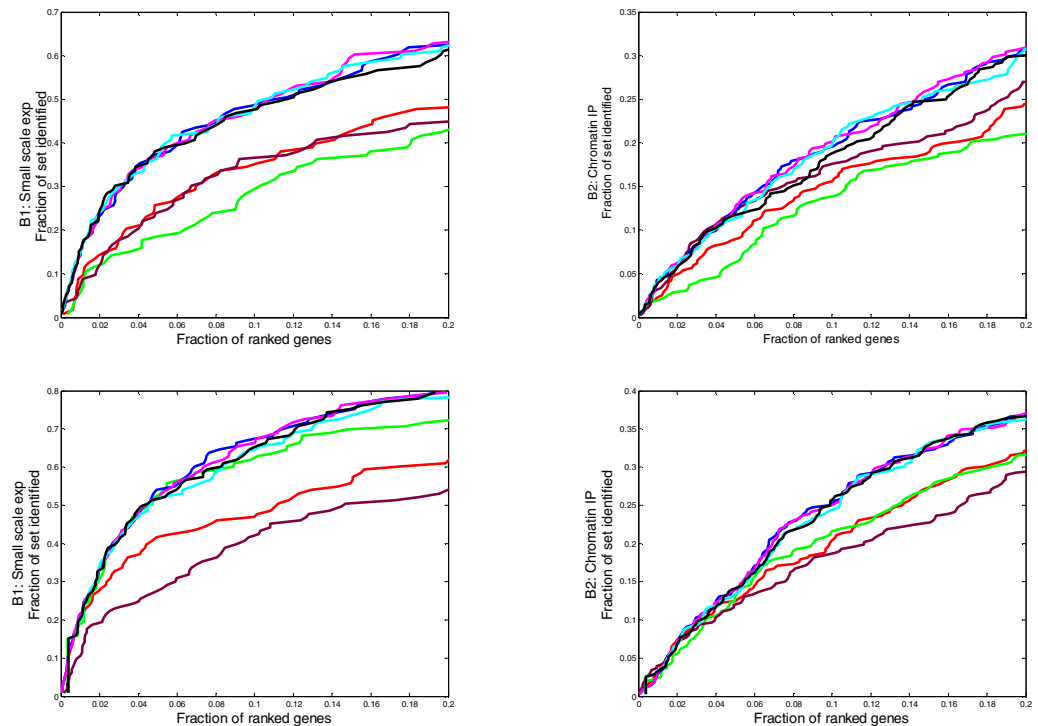

Fig. 1. Comparison of the three synchronization methods and the four $P$-value pooling methods. The fraction of the benchmark set that is identified is plotted as a function of the fraction of all the 5550 ranked genes. The different methods are colored as follows: alpha only (red), cdc15 only (green), cdc28 only (dark red), Fisher (blue), logit (magenta), Stouffer (cyan), and Liptak-stouffer (black). The graph on the left is based on the benchmark evaluation gene set $\mathrm{B} 1$, while the graph on the right is based on the gene set B2. Top row: results from Fisher's $g$ test. Bottom row: results from permutation method.

Table 1. Functional enrichment of periodically expressed genes identified by pooling the evidences with George and Mudholkar's logit method

\begin{tabular}{|l|l|l|l|}
\hline Organism & Enriched GO Term (total) & $\begin{array}{l}\text { Number of } \\
\text { periodic } \\
\text { genes in GO } \\
\text { term }\end{array}$ & $\begin{array}{l}\text { Significance of } \\
\text { Enrichment }\end{array}$ \\
\hline \multirow{5}{*}{ Budding } & M-phase (114) & 62 & 0.0062 \\
yeast & Cytokinesis (82) & 43 & 0.0429 \\
& Cytokinesis, contractile ring formation (10) & 9 & 0.0028 \\
& Cytokinesis, formation of actomyosin apparatus (10) & 9 & 0.0028 \\
& Chromosome condensation (11) & 9 & 0.0095 \\
& G1/S transition of mitotic cell cycle (20) & 13 & 0.0358 \\
\hline \multirow{5}{*}{ Fission } & M-phase (125) & 50 & 0.0155 \\
yeast & Cytokinesis (86) & 33 & 0.0761 \\
& Cytokinesis, completion of separation (5) & 4 & 0.0333 \\
& Cell separation during cytokinesis (5) & 4 & 0.0333 \\
& Chromosomal condensation (9) & 6 & 0.0281 \\
& G1/S transition of mitotic cell cycle (40) & 20 & 0.0079 \\
& G2/M transition of mitotic cell cycle (30) & 17 & 0.0027 \\
\hline
\end{tabular}


for the budding yeast when $\mathrm{q}$ is set to 0.05 . In a similar fashion, a list of 1315 genes was found to be cell cycle-regulated. These periodic genes were fed to GOMiner to find enriched GO terms. Table 1 shows the functional enrichment results.

\section{Conclusions}

In general, we are able to identify a better set of cell cycle-regulated genes by pooling the evidence from individual experiments. The performance of the four pooling methods was comparable. The performance of the Liptak-Stouffer method was slightly inferior to the other three pooling methods, while that of logit was marginally superior. While the Fisher's inverse Chi-square for pooling is quite popular, the other methods appeared to be less known.

In our functional enrichment studies, we found cell cycle-related GO terms were significantly enriched for the cell cycle-regulated genes identified using the Logit pooling method for both organisms. More interestingly, two children terms under cytokinesis term were more significantly enriched than the parent cytokinesis term for both organisms. It is also interesting to note that while there is an enrichment of genes from the G1/S transition process of the mitotic cell cycle in both organisms, genes from the G2/M transition process were significant only in $S$. pombe. It is also important to note that the study of data sets from two related organisms provided an opportunity to compare differences in significant genes for the cell cycle regulation.

We compared four different $P$-value pooling techniques and showed that they perform better than methods that use only a single data set. There are numerous potential applications for $P$-value pooling methods in the field of Bioinformatics. They are useful in any application where one has access to different data sets and statistical significance for the predictions.

\section{Acknowledgement}

Research of GN and EOG was supported in part by NIH Grant P01 DA15027-01.

\section{References}

1. Fisher, R.A., Statistical Methods for Research Workers. 14th ed. 1932, Edinburgh: Oliver and Boyd.

2. George, E.O. and G.S. Mudholkar, On the Convolution of Logistic Random Variables. Metrika, 1983. 30: p. 1-14.

3. Liptak, T., On the combination of independent tests. Magyar Tud. Akad. Mat. Kutato Int. Kozl, 1958. 3: p. 171-197.

4. Stouffer, S.A., E.A. Suchman, L.C. Devinney, S.A. Star, and R.M.J. Williams, The American Soldier, Vol. 1: Adjustment during army life. 1949, Princeton: Princeton University Press.

5. Bailey, T.L. and M. Gribskov, Combining evidence using p-values: application to sequence homology searches. Bioinformatics, 1998. 14(1): p. 48-54. 
6. Rustici, G., J. Mata, K. Kivinen, P. Lio, C.J. Penkett, G. Burns, J. Hayles, A. Brazma, P. Nurse, and J. Bahler, Periodic gene expression program of the fission yeast cell cycle. 2004. 36(8): p. 809-817.

7. Cho, R.J., M. Campbell, E. Winzeler, L. Steinmetz, A. Conway, L. Wodicka, T. Wolfsberg, A. Gabrielian, D. Landsman, D. Lockhart, and R. Davis, A genome-wide transcriptional analysis of the mitotic cell cycle. Molecular Cell, 1998. 2: p. 65-73.

8. Spellman, P.T., G. Sherlock, M.Q. Zhang, V.R. Iyer, K. Anders, M.B. Eisen, P.O. Brown, D. Botstein, and B. Futcher, Comprehensive Identification of Cell Cycle-regulated Genes of the Yeast Saccharomyces cerevisiae by Microarray Hybridization. Mol. Biol. Cell, 1998. 9(12): p. 3273-3297.

9. Oliva, A., A. Rosebrock, F. Ferrezuelo, S. Pyne, H. Chen, S. Skiena, B. Futcher, and J. Leatherwood, The Cell Cycle Regulated Genes of Schizosaccharomyces pombe. PLoS Biology, 2005. 3(7): p. e225.

10. Hartwell, L., J. Culotti, and B. Reid, Genetic control of the cell-division cycle in yeast. I. Detection of mutants. Proc Nat Acad Sci, 1970. 66: p. 352-359.

11. Johansson, D., P. Lindgren, and A. Berglund, A multivariate approach applied to microarray data for identification of genes with cell cycle-coupled transcription. Bioinformatics, 2003. 19(4): p. 467-473.

12. Zhao, L.P., R. Prentice, and L. Breeden, Statistical modeling of large microarray data sets to identify stimulus-response profiles. Proc. Natl. Acad. Sci., 2001. 98: p. 5631-5636.

13. Lu, X., W. Zhang, Z.S. Qin, K.E. Kwast, and J.S. Liu, Statistical resynchronization and Bayesian detection of periodically expressed genes. Nucl. Acids Res., 2004. 32(2): p. 447455.

14. Wichert, S., K. Fokianos, and K. Strimmer, Identifying periodically expressed transcripts in microarray time series data. Bioinformatics, 2004. 20(1): p. 5-20.

15. de Lichtenberg, U., L.J. Jensen, A. Fausboll, T.S. Jensen, P. Bork, and S. Brunak, Comparison of computational methods for the identification of cell cycle-regulated genes. Bioinformatics, 2005. 21(7): p. 1164-1171.

16. Bailey, T.L. and W.N. Grundy. Classifying proteins by family using the product of correlated p-values. in Proceedings of the Third international conference on computational molecular biology (RECOMB99). 1999.

17. Zeeberg, B.R., W. Feng, G. Wang, M.D. Wang, A.T. Fojo, M. Sunshine, S. Narasimhan, D.W. Kane, W.C. Reinhold, S. Lababidi, K.J. Bussey, J. Riss, J.C. Barrett, and J.N. Weinstein, GoMiner: A Resource for Biological Interpretation of Genomic and Proteomic Data. Genome Biology, 2003. 4(4): p. R28.

18. Benjamini, Y. and Y. Hochberg, Controlling the false discovery rate: a practical and powerful approach to multiple testing. Journal of the Royal Statistical Society, Series B, 1995. 57: p. 289-300. 